\title{
A comparison of batch mode and dynamic physiologically based bioaccessibility tests for PAHs in soil samples
}

\author{
Mark R Cave*, Joanna Wragg, Ian Harrison, Christopher H Vane
}

British Geological Survey, Keyworth, Nottingham, NG12 5GG, UK

\section{Tom Van de Wiele, Eva De Groeve}

LabMet, University of Ghent, Coupure Links 653, 9000 Ghent, Belgium

\section{C.Paul Nathanail, Matthew Ashmore}

University of Nottingham, School of Geography, Sir Clive Granger Building, The University of Nottingham, University Park, Nottingham, NG7 2RD, UK

\section{Russell Thomas, Jamie Robinson}

Parsons Brinckerhoff, Queen Victoria House, Redland Hill, Bristol, BS6 6US, UK

\section{Paddy Daly}

National Grid Property, Warwick Technology Park, Warwick, CV34 6DA, UK

\section{Abstract}

A fed state in vitro methodology capable of use in commercial testing laboratories has been developed for measuring the human ingestion bioaccessibility of polyaromatic hydrocarbons (PAHs) in soil (Fed ORganic Estimation human Simulation Test- FOREhST). The protocol for measuring PAHs in the simulated gastro-intestinal fluids used methanolic $\mathrm{KOH}$ saponification followed by a combination of polymeric sorbent solid phase extraction and silica sorbent cartridges for sample clean-up and preconcentration. The analysis was carried out using high pressure liquid chromatography with fluorescence detection. The repeatability 
1 of the method, assessed by the measurement of the bioaccessibility of 6 PAHs

2 (benz $[a]$ anthracene, benzo[b]fluoranthene, benzo[k]fluoranthene, benzo[ $[a]$ pyrene,

3 dibenz[ah]anthracene and indeno[1,2,3-c,d]pyrene) in eleven gas works soils, was c.10\%

4 RSD. The method compared well with the results from an independent dynamic human

5 simulation reactor comprising of the stomach, duodenal and colon compartments tested on

6 the same soils. The measured bioaccessible fraction of the soils varied from $10-60 \%$ for soils

7 containing 10-300 mg kg-1 $\mathrm{PAH}$ (the sum of the six studied) with total organic carbon

8 concentrations in the soils ranging from 1-13\%. A multiple regression model showed that the

9 PAH bioaccessible fraction could be explained using the PAH compound, the soil type and

10 the total PAH to soil organic carbon content. The method described here has potential for

11 site specific detailed quantitative risk assessment either to modify the risk estimation or to contribute to the risk evaluation.

13 Key words: PAH, soil, gas works, bioaccessibility, TOC, in vitro, FOREhST.

\section{Introduction}

Soil has been identified as the primary reservoir for PAH in the UK [1] and ingestion of soils is considered to be an important exposure pathway for humans [2]. For risk assessments considering the ingestion pathway, it is not the total PAH concentration that is important but the bioavailable fraction that enters the body. In this study, bioavailability is being discussed in terms of people ingesting contaminated soil. In this situation oral bioavailability of a given substance may be formally defined as the fraction of an administered dose that reaches the central (blood) compartment from the gastrointestinal tract [2]. This term should not be confused with the oral bioaccessibility, which is defined as the contaminant fraction of intake that is soluble in the human gastrointestinal system and is therefore available for absorption 
1 [2]. The methodologies used in this study estimate the bioaccessibility of PAHs using in

2 vitro physiologically based extraction methods.

3

4 The most commonly determined PAHs are the United States Environmental Protection

5 Agency list of sixteen "Consent Decree" priority pollutants [3, 4]. Of the sixteen, 6 benzo[a]pyrene (BaP) has been identified by the Environment Agency of England and Wales 7 (EA) as a non-threshold carcinogenic marker substance [5]. Soil Guideline Values (SGV) are 8 viewed as "trigger values", which are scientifically based generic assessment criteria (GAC), 9 for soil contamination, that are used to help evaluate the long to risks to human health.

Where soil concentrations exceed SGV, there may be a cause for concern to human health. Such levels may pose a significant risk to human health, although further investigation and evaluation of risks are required for the detailed risk assessment. No SGV for BaP has, to date, been issued by the EA for use in the UK, although a recent publication [3] has calculated GAC which are broadly equivalent to SGV values, of $0.83-2.1 \mathrm{mg} \mathrm{kg}^{-1}$ for BaP in residential and allotment soils varying in organic matter from $1 \%$ to $6 \%$; for other parts of Europe both the Danish and Flemish regulators have an intervention concentration/ cleanup directive value of $1 \mathrm{mg} \mathrm{kg}^{-1}[6,7]$.

For inorganic contaminants a number of in vitro bioaccessibility methods have been developed [8]. Importantly, since the bioaccessibility measurement from in vitro tests are methodologically defined, it is only useful in a risk assessment if it can be shown that the result is relevant to humans [9]. Soil arsenic bioaccessibility is now routinely considered in UK site specific detailed quantitative risk assessments (DQRA) (e.g.,[10-14]). The results from bioaccessibility tests are expressed as the bioaccessible fraction (BAF) as a percentage using the expression: 


$$
B A F(\%)=\left(\frac{\text { Element }_{\text {bioaccessible }}}{\text { Element }_{\text {total }}}\right) \times 100
$$

1

2 For inorganic bioaccessibility testing, evidence shows that the fasted state will give the most conservative estimate of the bioaccessible fraction as these give rise to lower $\mathrm{pH}$ conditions compared to the fed state [12-14]. A number of studies have shown that the presence of food increases the bioaccessibility of organic contaminants [15-17]. This effect is probably due to two separate influences. Food contains fat which can help mobilise hydrophobic organic contaminants into the aqueous solution and, when food is present in the human gastrointestinal (GI) tract, the amount of bile salts increases and these act as a surfactant, greatly reducing the surface tension of the digestive juice forming bile micelles with the organic contaminant $[16,17]$.

A number of studies have used in vitro bioaccessibility tests to estimate the human ingestion bioaccessibility of PAHs in soils. The German DIN standard bioaccessibility test [18], three Chinese studies used fasted conditions [17, 19, 20], the Dutch National Institute for Public Health and the Environment (RIVM) [21, 22], and Gron et al [6] have used the RIVM fed state model [22].

In a different approach, PAH release from a contaminated soil, containing 49 mg PAH $\mathrm{kg}^{-1}$, using a SHIME (Simulator of the Human Intestinal Microbial Ecosystem) reactor comprising the stomach, duodenal, and colon compartments [7] was investigated. The SHIME effectively models the human GI [23, 24]. The SHIME reactor differentiates itself from other in vitro intestinal models, because it is dynamic and comprises of the entire GI tract taking into account the enzymatic processes in the stomach and duodenum and the different characteristics of the microbiota along the colon reactors. 
1 Vasiluk et al [25] studied the role of the human stomach membrane as a sink for desorbed

$2 \mathrm{BaP}$ from a soil/sediment matrix. The authors made a case that a membrane sink should be considered in in vitro bioaccessibility testing suggesting that the driving force for uptake

4 from the soil is the fugacity gradient that exists between the gastrointestinal fluid and the 5 membrane.

6 Clearly, there are a number of experimental approaches to estimating the human 7 bioaccessibility of PAHs in soil samples, however, in order to produce comparative 8 reproducible and accurate data for risk assessments there needs to be a more standardised 9 approach to produce both comparable and validated data. To do this it is necessary to 10 develop a methodology which should be simple enough to be carried out by commercial 11 testing laboratories using standard laboratory equipment and analytical methodologies but retain enough complexity to still be a reasonable representation of the human GI tract. In the long term, this would involve the validation of the test against in vivo soil feeding trials and evaluation of the method in an inter-laboratory trial. The main aim of this study was to develop a standardised bioaccessibility test for PAHs in soils through the following objectives:

- Develop and /or adapt a suitable in vitro batch test for PAHs in soil with reference to the most up to date literature;

- Develop a robust analytical protocol for measuring PAHs in the simulated GI fluids arising from the in vitro batch test over a suitable concentration range for PAH

- Assess the repeatability of the test for a range of soil types containing varying concentrations of PAHs; 
- Compare the absolute bioaccessible fractions against a well established independent in vitro dynamic human GI simulator as guidance towards the potential of the batch method for simulating human bioavailability.

\section{$5 \quad$ Materials and methods}

6 Six PAHs were chosen based on their toxicity [26]. These were, in alphabetical order,

7 benz $[a]$ anthracene (BaA), benzo[b]fluoranthene $(\mathrm{BbF})$, benzo[k]fluoranthene (BkF),

8 benzo[a]pyrene BaP, Dibenz $[a, h]$ anthracene, DBA and indeno[1,2,3-c,d]pyrene (IP) .

$9 \quad$ Selected properties are shown in Table 1.

$10 \quad$ Table $1 \quad$ Selected PAH properties

\begin{tabular}{|c|c|c|c|c|c|c|c|}
\hline \multirow[t]{3}{*}{ PAH \& Abbreviation } & Relative & Number & $\mathrm{TEF}^{\mathrm{a}}$ & $\mathrm{PEF}^{\mathrm{c}}$ & Boiling & $\log K_{\mathrm{ow}}{ }^{\mathrm{d}}$ & EC \\
\hline & Molecular & of Rings & & \multicolumn{3}{|c|}{ Point } & $(\text { True })^{\mathrm{e}}$ \\
\hline & Mass & & & & ${ }^{o} \mathrm{C}^{\mathrm{d}}$ & & \\
\hline Benz$[a]$ anthracene, BaA & 228 & 4 & 0.1 & 0.145 & 435 & 5.91 & $25(18)$ \\
\hline Benzo[b]fluoranthene, BbF & 252 & 5 & 0.1 & 0.141 & 481 & 5.80 & $29(20)$ \\
\hline Benzo[k]fluoranthene, BkF & 252 & 5 & 0.1 & 0.1 & 481 & 6.00 & $29(20)$ \\
\hline Benzo $[a]$ pyrene, $\mathrm{BaP}$ & 252 & 5 & 1 & 1 & 496 & 6.04 & $30(20)$ \\
\hline Dibenz $[a, h]$ anthracene, DBA & 278 & 5 & $1^{\mathrm{b}}$ & 1.11 & 535 & 6.75 & $32(22)$ \\
\hline Indeno[1,2,3-c,d]pyrene, IP & 276 & 6 & 0.1 & 0.232 & 534 & 6.55 & $34(21)$ \\
\hline
\end{tabular}

$12{ }^{\mathrm{a}}$ Toxic Equivalent Factor (TEF), the toxicity of the compound expressed relative to benzo[a]pyrene [26, 27];

$13 \quad{ }^{\mathrm{b}}$ DBA TEF adopted from [27]

$14{ }^{\mathrm{c}}$ Potency Equivalent Factor (PEF) values used to derive the Land Quality Management (LQM)/Chartered 15 Institute of Environmental Health GAC, where PEF reflects the relative carcinogenic potency of the PAH

16 congeners rather than the differences in their general toxicity [3]

$17 \quad{ }^{\mathrm{d}}$ Boiling Point $\left({ }^{\circ} \mathrm{C}\right)$ and Octanol water partition coefficients (Kow) [28] 
1

2

4 Full experimental details of the analysis of the total organic carbon (TOC) content, the total

5 PAH content of the soil, the bioaccessibility extraction and subsequent analysis of PAH in the

6 extract using methanolic $\mathrm{KOH}$ saponification followed by a combination of polymeric 7 sorbent solid phase extraction and silica sorbent cartridges for sample clean-up and 8 preconcentration followed by analysis by high pressure liquid chromatography with 9 fluorescence detection is supplied in the Supplemental Information.

\section{Soil Samples}

11 Eleven soil samples were investigated. The soils were collected from disused gas works sites within the UK, and contained BaP concentrations ranging from 2 to $68 \mathrm{mg} \mathrm{kg}^{-1}$. The soils were prepared by first removing any large pieces of debris (stones, brick, pottery, plaster etc.), freeze dried, followed by retention of the fraction that passed through a $<250 \mu \mathrm{m}$ sieve. The $<250 \mu \mathrm{m}$ size fraction of soil was chosen as it is the fraction considered to be the upper limit of particle size that is likely to adhere to children's hands, who are often the at risk receptor for assessing contaminated sites [29] In addition, this fraction has been the dominant soil fraction in bioavailability and bioaccessibility studies over the last decade [19, 20, 30-33].

\section{In vitro Bioaccessibility Test}

The RIVM have carried out extensive literature reviews of the $\mathrm{pH}$, chemical environment and transit times for the human stomach and upper intestine and have developed fed and fasted bioaccessibility methods based on these findings [21, 22]. These methods have also been 
1 used successfully for organic contaminants in soil [6, 16, 34, 35]. It was therefore decided to

2 adopt the fed state RIVM method, with minor modifications, for this study. In order to distinguish this modified in vitro system from the RIVM method and other inorganic methodologies the method was named as the Fed ORganic Estimation human Simulation Test (FOREhST). The fed state was chosen as a number of studies have shown that for hydrophobic organics the fed state gives the most conservative estimate of bioaccessibility [15-17]. The RIVM study used an infant formula supplemented with vegetable oil as the food component; This food component was chosen to represent an average diet for men and women aged 19-65 in the Netherlands, with respect to their mean intake of energy and nutrients [22]. Initial work in this study used an infant formula which, for routine use, was found to be difficult to weigh out into the extraction tubes and, once opened, did not last long before it degraded and was not suitable for use. The wet food product was replaced with a freeze dried oatmeal and rice porridge infant food supplemented with sunflower oil to match the macronutrient composition of the average diet of 4-6 year old children in Britain [36]. The FOREhST method is essentially a three stage static in vitro bioaccessibility test, which is intended to simulate the physico-chemical conditions in the fed state. The method is carried out at human body temperature $\left(37^{\circ} \mathrm{C}\right)$ and utilises end-over-end rotation. The stages involved in the methodology are suggestive of the saliva, gastric and intestinal (duodenal and bile) phases of the human gastro-intestinal system, with sample collection (by centrifugation) at the end of the extraction phase representative of small intestinal digestion. Gastrointestinal fluid $\mathrm{pH}$, ratios and transit times are all adjusted, compared to a fasted static model, to account for the physiological differences caused by the ingestion of food: saliva $\mathrm{pH}(6.8 \pm$ 0.5); gastric $\mathrm{pH}(1.3 \pm 0.5$ ); small intestinal $\mathrm{pH}$ (duodenal $\mathrm{pH} 8.1 \pm 0.2$, bile $\mathrm{pH} 8.2 \pm 0.2$ ); GI fluid ratio for saliva: gastric: duodenal: bile(1:2:2:1), GI transit time (gastric $2 \mathrm{hr}$, small intestine $2 \mathrm{hr}$ ). For each contaminated soil under investigation, $0.3 \mathrm{~g}$ of contaminated material 
1 was extracted in triplicate, with extraction of each replicate on consecutive days in order to

2 estimate the repeatability of the method. Sample blanks were extracted within each batch of

3 samples under investigation. A detailed description of the FOREhST bioaccessibility test can

4 be found in the Supporting Information.

\section{The SHIME method comparison}

6 The results of the FOREhST method were compared to the SHIME in vitro GI model [7, 23,

7 24]. Each of the eleven soils were run in triplicate using the SHIME system with the same

8 food component used in the FOREhST test. Full details of the conditions used for SHIME

9 model are given in the supplementary information. The stomach and intestine extracts from

10 the SHIME reactor were analysed for their PAH content using the same method used for the

11 FOREhST extracts.

\section{Results and Discussion}

\section{Performance of the analytical method}

14 The performance of the test on spiked portions of the blank extraction solutions from both the

15 FOREhST and SHIME methods was shown to be good: recoveries of c.90\% or better with

16 repeatability relative standard deviations of c. $5 \%$ or better (Table 2).

17 Table 2 Solid phase extraction recoveries of 6 PAHs from various spiked saponified

18 G.I fluids

\begin{tabular}{ccccccccc} 
G.I & \multicolumn{2}{c}{ No G.I Fluid } & \multicolumn{2}{c}{ FOREhST Fed } & \multicolumn{2}{c}{ SHIME Fed } & \multicolumn{2}{c}{ SHIME Fasted } \\
PAH & Av. \% & RSD & Av. \% & RSD & Av. \% & RSD & Av. \% & RSD \\
& & & & & & & & \\
BaA & 102.6 & 4.6 & 90.8 & 5.7 & 92.0 & 1.3 & 89.6 & 2.5 \\
BbF & 97.0 & 2.7 & 91.1 & 2.7 & 95.5 & 0.6 & 93.0 & 2.2 \\
BkF & 95.5 & 2.0 & 90.8 & 1.9 & 93.8 & 0.5 & 93.0 & 2.2 \\
BaP & 96.6 & 3.5 & 88.0 & 3.7 & 92.3 & 1.2 & 89.7 & 2.8 \\
DBA & 89.3 & 1.5 & 86.6 & 3.2 & 92.6 & 1.1 & 88.6 & 3.5 \\
IP & 89.1 & 1.4 & 89.2 & 2.2 & 90.5 & 1.1 & 89.4 & 3.5
\end{tabular}




\section{Comparison of the SHIME and FOREhST bioaccessibility results}

2 For the FOREhST method the majority of the samples have RSD values of $10 \%$ or better and

3 the SHIME method $15 \%$ or better.

4 Since there is uncertainty associated with both measurements it is not appropriate to use

5 ordinary least squares regression. The weighted total least squares (WTLS) method which

6 takes account of the error on both sets of data [37] and Theil's non-parametric method that

7 makes no assumptions about distributions of errors and is robust to outliers have been used to

8 assess the linear relationship between the two measurements [38, 39].

9 Table 3 Summary of the linear regression parameters for the SHIME FOREhST data

10 comparison

$\begin{array}{ccccccc}\text { PAH } & \begin{array}{c}\text { WTLS } \\ \text { Slope }\end{array} & \begin{array}{c}\text { WTLS } \\ \text { Intercept }\end{array} & \begin{array}{c}\text { Theil } \\ \text { Slope }\end{array} & \begin{array}{c}\text { Theil } \\ \text { Intercept }\end{array} & \mathrm{r} & \text { p value } \\ \mathrm{BaA} & 0.35 & 26.0 & 0.28 & 31.7 & 0.36 & 0.275 \\ \mathrm{BbF} & 0.53 & 18.8 & 0.51 & 20.7 & 0.51 & 0.110 \\ \mathrm{BkF} & 0.49 & 14.1 & 0.72 & 11.2 & 0.53 & 0.093 \\ \mathrm{BaP} & 0.74 & 9.3 & 0.65 & 14.6 & 0.65 & 0.029 \\ \mathrm{DBA} & 0.72 & 4.7 & 0.82 & 3.5 & 0.71 & 0.015 \\ \mathrm{IP} & 0.71 & 14.0 & 0.65 & 17.1 & 0.68 & 0.021 \\ \text { All PAHs } & 0.85 & 3.9 & 0.87 & 8.1 & 0.74 & <0.001\end{array}$

11 

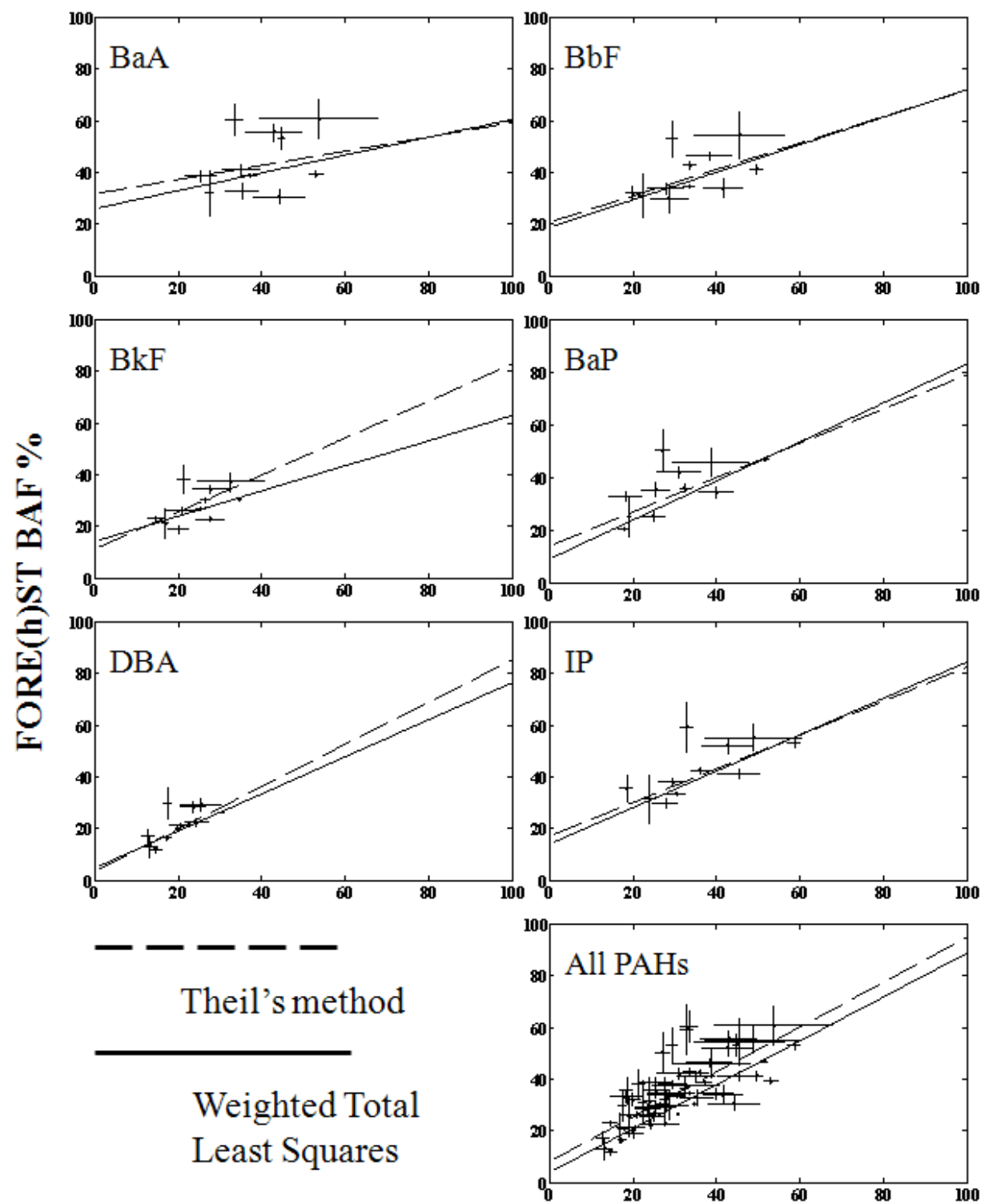

SHIME BAF \%

2 Figure 1 Comparison of the BAF for the SHIME and the FORE(h)ST methods

3 The slope and intercepts for both methods and Pearson correlation coefficients (r) and their

4 associated significance are given in Table 3 and the scatter plots in Figure 1 . There is

5 reasonable agreement between the two regression methods. Although the original samples

6 were chosen to have a range of total PAH (BaP ranges from c.2 to c. $70 \mathrm{mg} \mathrm{kg}^{-1}$ ) the range of

7 BAF for each individual PAH is relatively low, with BAF of the individual PAHs covering

$830 \%$ or less of the total range of concentrations in the soil. This may account for the 
1 relatively low r values and variability in slope for individual PAHs compared to the combined

2 data set. When all PAHs are considered together, there is highly a significant correlation

3 with a strong $\mathrm{r}$ value of 0.74 (Table 3 ). There is however a trend towards increasing $\mathrm{r}$ values

4 with increasing hydrophobicity (cf Table $1 \mathrm{~K}_{\mathrm{ow}}$ and Table $3 \mathrm{r}$ ). BaA has a weak non-

5 significant correlation (0.36); Bbf and Bkf have moderate correlations (0.51 and 0.53) which

6 are significant at the $90 \%$ confidence level; $\mathrm{BaP}$, IP and DBA have strong correlations (0.65,

$7 \quad 0.68$ and 0.71 ) which are significant the 95\% confidence level. There is not enough data (the

8 variety of soils and PAH sources in this study are limited and the agreement when all PAHs

9 are considered is good) to conclude that the two methods do not agree for the least

10 hydrophobic PAHs. The trend, however, suggests that there is more variability in the BAF for low Kow PAHs. A possible explanation for this is that PAH extraction from the soil is through extraction into micelles which are formed in the GI extraction medium [22]. Research into the solubility of PAH in micelles [40] suggests that the more hydrophobic compounds are held in the centre of the micelle whereas the less hydrophobic compounds are solubilised at the interfacial medium. Therefore, during the in vitro extraction test used here, once the hydrophobic PAHs are released from the soil they are held within the centre of the micelle away from the soil surface but the less hydrophobic compounds at the surface of the micelle can interact with the soil and be redistributed back onto the soil leading to a greater variability the measured BAF.

Whilst Figure 1 shows the individual FOREhST BAF values for each PAH in each soil are not always higher than the SHIME values, the slopes for the regression lines (Table 3) for all individual PAHs and all PAHs combined together are all below unity showing that on average the FOREhST method gives higher BAF than the SHIME method. 
1 Taking all PAHs together slope of the line suggests that the SHIME method BAF values are c. $80 \%$ of the FOREhST values showing that the FOREhST method over predicts compared to the dynamic SHIME model.

The variability in BAF results between the soils in this study and PAHs is likely to be derived from three sources:

i) The physico-chemical properties of the soils;

ii) The physico-chemical properties of the PAHs; and

iii) The original total concentration of PAH in the soil.

With respect to point iii) the mechanism for PAH sorption to soils is complex [41], with multistage sequestration on to humic and fulvic acid polymer layers as well as adsorption on to the soil mineral surfaces [42]. The different sorption sites on the soil will have different PAH affinity and absolute capacity [41] depending on the make-up of the soil. The overall PAH availability will therefore be a function of how much PAH is sorbed to the soil and how tightly it binds to the different sorption sites on the soil surface.

The organic matter content of soils is often reported to be positively correlated with total PAH content [43] which is related to the sorption capacity of the organic carbon in the soil. Since there are varying concentrations of both TOC and PAHs in the soils under test the ratio of the individual PAH concentration to the soil TOC $\left(\mathrm{mg} \mathrm{g}^{-1}\right)$ was used to standardise the amount of PAH relative to the TOC.

To understand how these parameters affect the final results, a simple linear regression model was set up using the BAF value of the FOREhST method as the dependant variable (the Y variable) and the soil sample, PAH molecule and the ratio of the original PAH concentration to the TOC concentration ( $\mathrm{mg} \mathrm{g}^{-1}$ ) as the independent variables (the $\mathrm{X}$ variables). Since soil and PAH molecule are factors rather than continuous variables the regression was set up so 
1 that the regression equation was relative to the soil with the lowest TOC (Soil 1) and the

2 lowest molecular weight $\mathrm{PAH}(\mathrm{BaA}$, Table 1). The regression coefficients are given in Table

3 4. The equation of the model is:

$$
B A F=\beta_{0}+\beta P A H \text { molecule }+\beta \text { Soil }+\beta_{1} \times\left(\frac{\text { total PAH conc }}{\text { TOC }}\right)
$$

For example, referring to Table 4, the predicted BAF for the PAH BbF in Soil 2 would be:

$$
B A F_{B b F_{\text {, soil } 2}}=35.8-5.3-1.77+12.2 \times \frac{\text { total } B \text { bF in Soil } 2}{\text { TOC in Soil2 }}
$$

The model accounts for c. 90\% of the variance in the data (Multiple R-squared: 0.94, Adjusted R-squared: 0.92). All of the parameters, apart from the effect of the PAH IP and soils 2, 4 and 8, are significant. In words, the model shows that on average c. 36\% (intercept) of the PAH is bioaccessible. The coefficients associated with the factors show how much the average value is adjusted for specific PAHs and soils. The higher molecular weight PAHs have reduced bioaccessibility relative to $\mathrm{BaA}$ (negative coefficients ranging from -5.3 to 16.9\%, Table 4) apart from IP which is not significantly different from BaA (p value of 0.745, Table 4). Apart from IP the PAH coefficients indicate reduction of the BAF with log $\mathrm{K}_{\mathrm{ow}}$ (see Table 1) i.e the higher the hydrophobicity the lower the amount of PAH extracted by the in vitro GI simulation test which is in general agreement with other bioaccessibility studies [17, 19]. IP, which has the second highest log Kow has a coefficient which is indistinguishable from $\mathrm{BaA}$ which has the lowest value. The reason for this is not clear since the literature does not show that IP sorption to soils shows any anomalous effects. The simulated stomach and intestine fluids for the SHIME and FOREhST methods contain a complex mixture of organic reagents and in the case of IP it appears that there is another property of IP besides log Kow which is governing its extraction in this matrix. The biological activity of PAHs in specific situations can be shown to be related to the shape of the molecule as defined by topographical properties e.g. [44] using a Quantitative Structure 
1 Activity Relationship (QSAR) approach. The Pearson correlation coefficient between the

2 coefficients for the PAHs (Table 4) and the Kow of the PAHs (Table 1) is weak (-0.29) but in

3 a list of molecular descriptors for PAHs [45], there is a strong correlation (0.70) between the

4 PAH coefficients and a topological descriptor called DECC which is an eccentricity index

5 measuring the size and shape of the molecule. As only 6 PAHs have been investigated in this

6 work there is not enough data to reach any conclusions regarding the anomalous IP

7 behaviour, and a further study using a larger suite of PAHs would be required to test whether

8 DECC or other properties are involved in the extraction mechanism.

9 Table 4 Linear regression coefficients and associated statistics for the BAF MLR

10 model

$\begin{array}{ccccc}\text { Coefficient name } & \text { Independent Parameter } & \text { Coefficient value } & \text { Std. Error } & \text { p value } \\ \beta_{0} & \text { Intercept } & 35.8 & 1.9 & <0.001 \\ \beta_{\text {BaA }} & \text { BaA } & 0 & - & - \\ \beta_{\mathrm{BbF}} & \text { BbF } & -5.3 & 1.5 & <0.001 \\ \beta_{\mathrm{BkF}} & \mathrm{BkF} & -13.5 & 1.6 & <0.001 \\ \beta_{\text {BaP }} & \text { BaP } & -9.0 & 1.6 & <0.001 \\ \beta_{\mathrm{DBA}} & \text { DBA } & -16.9 & 2.2 & <0.001 \\ \beta_{\mathrm{IP}} & \text { IP } & 0.50 & 1.5 & 0.745 \\ \beta_{\text {Soil1 }} & \text { Soil 1 } & 0 & - & - \\ \beta_{\text {Soil2 }} & \text { Soil 2 } & -1.77 & 2.0 & 0.371 \\ \beta_{\text {Soil3 }} & \text { Soil 3 } & 12.6 & 2.0 & <0.001 \\ \beta_{\text {Soil4 }} & \text { Soil 4 } & -4.1 & 2.8 & 0.138 \\ \beta_{\text {Soil5 }} & \text { Soil 5 } & 1.1 & 2.6 & 0.684 \\ \beta_{\text {Soil6 }} & \text { Soil 6 } & -8.1 & 2.2 & <0.001 \\ \beta_{\text {Soil7 }} & \text { Soil 7 } & -8.0 & 2.1 & <0.001 \\ \beta_{\text {Soil8 }} & \text { Soil 8 } & -2.5 & 2.2 & 0.254 \\ \beta_{\text {Soil9 }} & \text { Soil 9 } & 6.4 & 2.1 & 0.004 \\ \beta_{\text {Soil10 }} & \text { Soil 10 } & 9.6 & 2.9 & 0.002 \\ \beta_{\text {Soil11 }} & \text { Soil 11 } & 10.1 & 3.1 & 0.002 \\ \beta_{1} & \text { PAH/TOC ratio } & 12.2 & 0.35 & 0.001\end{array}$

11

12 The soils show varying increases and decreases in bioaccessibility relative to soil 1 13 (coefficients ranging from -8.1 to $12.6 \%$ ). The coefficient for the PAH to TOC ratio also has 14 a highly significant effect ( $p$ value $=0.001$, Table 4 ). To give an idea of scale for this parameter, using $\mathrm{BaP}$ in soil 5 as an example, an increase in the $\mathrm{BaP}$ concentration of $14 \mathrm{mg}$ 
$1 \mathrm{~kg}^{-1}$ increases the modelled BAF by $5 \%$ (from $39 \%$ to $44 \%$ ) and increasing the TOC

2 concentration by 3\% organic carbon decreases the BAF by 5\% (from 39\% to 34\%)..

3 Similar regression results were obtained when the SHIME BAF replaced the FOREhST as

4 the independent variable in the linear model (data not shown). Despite the inclusion of the

5 PAH to TOC ratio in the model the effect of the different soils was also significant suggesting

6 that there are other soil properties which also control the BAF.

7 Although there have been a number of important findings arising from this study they cannot

8 be considered as generic until the methodology has been applied to a wider variety of soil

9 types and contamination sources as well as extending the PAHs investigated.

10 From the method development stand point the study has met the four objectives outlined in

11 the introduction as follows:

- The FOREhST method can be set up and used in any competent testing laboratory;

- The analysis of the PAHs in the simulated GI fluids is robust and accurate (Table 2);

- The bioaccessibility results for the soils studied were repeatable (mostly $<10 \%$ RSD);

- The FOREhST method gives comparable results to the dynamic SHIME human GI model giving some assurance that the method is likely to have some relevance to human bioavailability; and

- The FOREhST method gives conservative results (c.20\% higher) compared to the SHIME method.

From a risk estimation standpoint the bioaccessibility data produced from this test could not be used on their own as an input to a DQRA to derive site specific assessment criteria as further validation tests are required which include:

i) Testing the applicability of the method on a wider variety of soil types and PAHs; 
ii) Assessing the reproducibility of the method through an inter-laboratory trial;

iii) Validation of the results against in-vivo trials;

iv) The production of PAH reference soils to allow performance of the method to be checked and monitored using standard QA/QC protocols;

Despite this, even at this early stage, bioaccessibility data from this test could be used to provide information in a "lines of evidence approach" to aid the risk evaluation stage of assessment.

\section{Interpretation of the bioaccessibility results}

From the point of view of risk assessment, taking into account that the study was carried out on a limited range of soils, the fact that a quantitative model predicting BAF using soil and PAH properties was achieved has the following consequences:

i) For well characterised sites it may be feasible to calculate on a site specific basis the PAH BAF for a number of PAHs based on the measurements of a few "marker" compounds;

ii) Quantitative data on the effect of different PAH to TOC ratios on the BAF factor can provide invaluable information when regulators or developers are deriving site specific assessment criteria for PAHs in soil; and

iii) The MLR model gives valuable insights into how the physico-chemical parameters governing the soil/PAH system control the BAF and hence help to optimise both risk evaluation and soil remediation strategies.

Acknowledgements 
1 This paper is published with permission of the Executive Director of the British Geological

2 Survey. The project was funded by National Grid and project managed by Parsons

3 Brinkerhoff.

\section{Supporting Information Available}

Methods used for total organic carbon analysis and total PAH content of the soils, the operating conditions for the SHIME method, the extraction and subsequent analysis of the PAHs from the FOREhST method, tables containing the total PAH content of the soils and the comparative bioaccessibility factors for all PAHs in all soils. This information is available free of charge via the Internet at http://pubs.acs.org.

\section{References}

1. Menzie, C. A.; Potocki, B. B.; Santodonato, J., Exposure to Carcinogenic PAHs in the Environment. Environmental Science \& Technology 1992, 26, (7), 1278-1284.

2. Paustenbach, D. J., The practice of exposure assessment: A state-of-the-art review (Reprinted from Principles and Methods of Toxicology, 4th edition, 2001). J. Toxicol. Env. Health-Pt b-Crit. Rev. 2000, 3, (3), 179-291.

3. $\quad$ Nathanail, P.; McCaffrey, C.; Ashmore, M.; Cheng, Y.; Gillet, A.; Ogden, R.; Scott, D., The LQM/CIEH Generic Assessment Criteria for Human Health Risk Assessment 2nd ed.; Land Quality Press: Nottingham, 2009.

4. UNITED STATES ENVIRONMENTAL PROTECTION AGENCY Quality criteria for water. ; EPA 440/5-86-001.; United States Environmental Protection Agency, Washington DC, USA.: 1987.

5. Department for the Environment Food and Rural Affairs and the Environment Agency Assessment of Risks to Human Health from Land Contamination: An Overview of the Development of Guideline Values and related Research; CLR7; 2002.

6. $\quad$ Gron, C.; Oomen, A.; Weyand, E.; Wittsiepe, J., Bioaccessibility of PAH from Danish soils. Journal of Environmental Science and Health Part a-Toxic/Hazardous Substances \& Environmental Engineering 2007, 42, (9), 1233-1239.

7. Van de Wiele, T. R.; Verstraete, W.; Siciliano, S. D., Polycyclic aromatic hydrocarbon release from a soil matrix in the in vitro gastrointestinal tract. Journal of Environmental Quality 2004, 33, (4), 1343-1353.

8. Wragg, J.; Cave, M. R. In-vitro Methods for the Measurement of the Oral Bioaccessibility of Selected Metals and Metalloids in Soils: A Critical Review; R\&D Project Record P5-062/TR/01; Environment Agency: 2003.

9. Nathanail, C. P. Professional Practice Note: Reviewing Human Health Risk Assessment Reports Invoking Contaminant Oral Bioavailability Measurements Or Estimates. www.cieh.org.uk 
10. Nathanail, C. P.; McCaffrey, C.; Haynes, D., Assessing exposure to pedogenic arsenic contamination at a dwelling in Northamptonshire, UK: a case study. Soil Use Manage. 2005, 21, (0), 508-517.

11. Nathanail, C. P.; Smith, R., Incorporating bioaccessibility in detailed quantitative human health risk assessments. . J. Environ. Sci. Health, Part A 2007, 42, (9), 1193-1202.

12. Maddaloni, M.; Lolacono, N.; Manton, W.; Blum, C.; Drexler, J.; Graziano, J., Bioavailability of soilborne lead in adults, by stable isotope dilution. Environmental Health Perspectives 1998, 106, 1589-1594.

13. Oomen, A. G.; Hack, A.; Minekus, M.; Zeijdner, E.; Cornelis, C.; Schoeters, G.; Verstraete, W.; Van de Wiele, T.; Wragg, J.; Rompelberg, C. J. M.; Sips, A.; Van Wijnen, J. H., Comparison of five in vitro digestion models to study the bioaccessibility of soil contaminants. Environmental Science \& Technology 2002, 36, (15), 3326-3334.

14. Van de Wiele, T.; Boon, N.; Possemiers, S.; Jacobs, H.; Verstraete, W., Inulin-type fructans of longer degree of polymerization exert more pronounced in vitro prebiotic effects. Journal of Applied Microbiology 2007, 102, (2), 452-460.

15. Hack, A.; Selenka, F., Mobilization of PAH and PCB from contaminated soil using a digestive tract model. Toxicol. Lett. 1996, 88, (1-3), 199-210.

16. Oomen, A. G.; Sips, A.; Groten, J. P.; Sijm, D.; Tolls, J., Mobilization of PCBs and lindane from soil during in vitro digestion and their distribution among bile salt micelles and proteins of human digestive fluid and the soil. Environmental Science \& Technology 2000, 34, (2), 297-303. 17. Tang, X. Y.; Tang, L.; Zhu, Y. G.; Xing, B. S.; Duan, J.; Zheng, M. H., Assessment of the bioaccessibility of polycyclic aromatic hydrocarbons in soils from Beijing using an in vitro test. Environmental Pollution 2006, 140, (2), 279-285.

18. DIN Soil Quality - Absorption availability of organic and inorganic pollutants from contaminated soil material; DIN E 19738; Deutsches Institut fur Normung e. V.: Berlin, 2000. 19. Khan, S.; Cao, Q.; Lin, A. J.; Zhu, Y. G., Concentrations and bioaccessibility of polycyclic aromatic hydrocarbons in wastewater-irrigated soil using in vitro gastrointestinal test. Environmental Science and Pollution Research 2008, 15, (4), 344-353.

20. Lu, M.; Yuan, D.; Lin, Q.; Ouyang, T. Assessment of the bioaccessibility of polycyclic aromatic hydrocarbons in topsoils from different urban functional areas using an in vitro gastrointestinal test. http://dx.doi.org/10.1007/s10661-009-0982-x

21. Sips, A. J. A. M.; Bruil, M. A.; Dobbe, C. J. G.; Van de Kamp, E.; Oomen, A. G.; Pereboom, D. P. K. H.; Rompelberg, C. J. M.; Zeilmakker, M. J. Bioaccessibility of Contaminants form Ingested Soil in Humans; 711701012 RIVM: 2001.

22. Versantvoort, C. H. M.; van de Kamp, E.; Rompelberg, C. J. M. Development and applicability of an in vitro digestion model in assessing the bioaccessibility of contaminants from food; 3201020022004; RIVM: 2004.

23. Molly, K.; Vandewoestyne, M.; Desmet, I.; Verstraete, W., Validation of the Simulator of the Human Intestinal Microbial Ecosystem (Shime) Reactor Using Microorganism-Associated Activities. Microb. Ecol. Health Dis. 1994, 7, (4), 191-200.

24. Molly, K.; Woestyne, M. V.; Verstraete, W., Development of a 5-Step Multichamber Reactor as a Simulation of the Human Intestinal Microbial Ecosystem. Applied Microbiology and Biotechnology 1993, 39, (2), 254-258.

25. Vasiluk, L.; Pinto, L. J.; Walji, Z. A.; Tsang, W. S.; Gobas, F.; Eickhoff, C.; Moore, M. M., Benzo[a]pyrene bioavailability from pristine soil and contaminated sediment assessed using two in vitro models. Environmental Toxicology and Chemistry 2007, 26, (3), 387-393.

26. Nisbet, I. C. T.; Lagoy, P. K., Toxic Equivalency Factors (TEFs) for Polycyclic AromaticHydrocarbons (PAHs) Regulatory Toxicology and Pharmacology 1992, 16, (3), 290-300.

27. Malcolm, H. M.; Dobson, S. The calculation of an environmental assessment level (EAL) for atmospheric PAHs using relative potencies.; Report No. DoE/HMIP/RR/94/041 Department of the Environment: London, UK., 1994; pp pp. 34-46.

28. Alves de Lima Ribeiro, F.; Ferreira, M. M. C., QSPR models of boiling point, octanol-water partition coefficient and retention time index of polycyclic aromatic hydrocarbons. Journal of Molecular Structure: THEOCHEM 2003, 663, (1-3), 109-126. 
29. Duggan, M. J.; Inskip, M. J.; Rundle, S. A.; Moorcroft, J. S., Lead in Playground Dust and on the Hands of Schoolchildren. Sci. Total Environ. 1985, 44, (1), 65-79.

30. $\quad$ Casteel, S. W.; Weis, C. P.; Henningsen, G. M.; Brattin, W. J., Estimation of Relative Bioavailability of Lead in Soil and Soil-Like Materials Using Young Swine. Environmental Health Perspectives 2006, 114, (8), 1162-1171.

31. Kelley, M. E.; Brauning, S. E.; Schoof, R.; Ruby, M. V., Assessing Oral Bioavailability of Metals in Soil. Battelle Press: Columbus Richland, 2002.

32. Schroder, J. L.; Basta, N. T.; Casteel, S. W.; Evans, T. J.; Payton, M. E.; Si, J., Validation of the in vitro gastrointestinal (IVG) method to estimate relative bioavailable lead in contaminated soils. Journal Of Environmental Quality 2004, 33, (2), 513-521.

33. Tang, J. X.; Petersen, E. J.; Huang, Q. G.; Weber, W. J., Development of engineered natural organic sorbents for environmental applications: 3. Reducing PAH mobility and bioavailability in contaminated soil and sediment systems. Environmental Science \& Technology 2007, 41, (8), 29012907.

34. Hansen, J. B.; Oomen, A. G.; Edelgaard, I.; Gron, C., Oral bioaccessibility and leaching: Tests for soil risk assessment. Engineering in Life Sciences 2007, 7, (2), 170-176.

35. Hurdzan, C. M.; Basta, N. T.; Hatcher, P. G.; Tuovinen, O. H., Phenanthrene release from natural organic matter surrogates under simulated human gastrointestinal conditions. Ecotoxicology and Environmental Safety 2008, 69, (3), 525-530.

36. $\quad$ Gregory, J.; Lowe, S.; Bates, C. J.; Prentice, A.; Jackson, L. V.; Smithers, G.; Wenlock, R.; Farron, N., National Diet and Nutrition Survey :young people aged 4 to 18 years. In Agency, F. S., Ed. The Stationery Office: London, 2000; Vol. Volume 1: Report of the diet and nutrition survey. 37. Krystek, M.; Anton, M., A weighted total least-squares algorithm for fitting a straight line. Measurement Science and Technology 2007, 18, 3438-3442.

38. Glaister, P., Robust linear regression using Theil's method. Journal of Chemical Education 2005, 82, (10), 1472-1473.

39. Theil, H., A rank-invariant method of linear and polynomial regression analysis. Indagationes Mathematicae 1950, 12, 85-91.

40. Lan Chun, C.; Lee, J.-J.; Park, J.-W., Solubilization of PAH mixtures by three different anionic surfactants. Environmental Pollution 2002, 118, (3), 307-313.

41. Haritash, A. K.; Kaushik, C. P., Biodegradation aspects of Polycyclic Aromatic Hydrocarbons (PAHs): A review. Journal of Hazardous Materials 2009, 169, (1-3), 1-15.

42. Luo, L.; Zhang, S. Z.; Ma, Y. B., Evaluation of impacts of soil fractions on phenanthrene sorption. Chemosphere 2008, 72, (6), 891-896.

43. Nam, J. J.; Sweetman, A. J.; Jones, K. C., Polynuclear aromatic hydrocarbons (PAHs) in global background soils. Journal of Environmental Monitoring 2009, 11, (1), 45-48.

44. Benísek, M.; Bláha, L.; Hilscherová, K., Interference of PAHs and their N-heterocyclic analogs with signaling of retinoids in vitro. Toxicology in Vitro 2008, 22, (8), 1909-1917.

45. Todeschini, R.; Gramatica, P.; Marengo, E.; Provenzani, R., Weighted Holistic Invariant Molecular Descriptors. Part 2. Theory Development and Applications on Modeling Physico-Chemical Properties of PolyAromatic Hydrocarbons (PAH). . Chemometics and Intelligent Laboratory Systems 1995, 27, 221-229. 


\section{Brief}

2 The performance of a batch mode and a dynamic in vitro test for estimating the human 3 ingestion bioaccessibility of PAHs in soils are compared. 